\title{
Positive solutions of nonhomogeneous boundary value problems for some nonlinear equation with $\phi$-Laplacian
}

Liang-Gen $\mathrm{Hu}^{*}$ and Jing $\mathrm{Xu}$

\section{*Correspondence:}

hulianggen@yahoo.cn

Department of Mathematics,

Ningbo University, Ningbo, 315211, P.R. China

\section{囪 Springer}

\begin{abstract}
We will consider the nonhomogeneous $\phi$-Laplacian differential equation

$$
\left\{\begin{array}{lc}
\left(\phi\left(u^{\prime}(t)\right)\right)^{\prime}=-h(t) f(u(t)), & t \in(0, T), \\
u(0)=\sum_{i=1}^{k} \alpha_{i} u\left(\eta_{i}\right), & \phi\left(u^{\prime}(T)\right)=\beta,
\end{array}\right.
$$

where $\phi: \mathbb{R} \rightarrow(-b, b)(0<b \leq+\infty)$ is an increasing homeomorphism such that $\phi(0)=0, h:[0, T] \rightarrow \mathbb{R}^{+}$and $f: \mathbb{R}^{+} \rightarrow \mathbb{R}^{+}$are continuous, $\beta \geq 0$ and $\eta_{i} \in(0, T)$ and $\alpha_{i} \in \mathbb{R}, i=1,2, \ldots, k$. Based on the Krasnosel'skii fixed point theorem, the existence of a positive solution is obtained, even if some of the $\alpha_{i}$ coefficients are negative. Two examples are also given to illustrate our main results.
\end{abstract}

Keywords: nonhomogeneous; $\phi$-Laplacian; positive solution; fixed point; negative coefficient

\section{Introduction}

We are concerned with the $\phi$-Laplacian differential equation with the nonhomogeneous Dirichlet-Neumann boundary conditions

$$
\left\{\begin{array}{l}
\left(\phi\left(u^{\prime}(t)\right)\right)^{\prime}=-h(t) f(u(t)), \quad t \in(0, T), \\
u(0)=\omega(u):=\sum_{i=1}^{k} \alpha_{i} u\left(\eta_{i}\right), \quad \phi\left(u^{\prime}(T)\right)=\beta,
\end{array}\right.
$$

where $\phi: \mathbb{R} \rightarrow(-b, b)(0<b \leq+\infty)$ is an increasing homeomorphism such that $\phi(0)=0$, $h:[0, T] \rightarrow \mathbb{R}^{+}$and $f: \mathbb{R}^{+} \rightarrow \mathbb{R}^{+}$are continuous $\left(\mathbb{R}^{+}=[0,+\infty)\right), \beta \geq 0, \alpha_{i} \in \mathbb{R}$ and $\eta_{i} \in$ $(0, T)$, for $i \in I:=\{1,2, \ldots, k\}$.

Boundary value problems, including the $\phi$-Laplacian operator, have received a lot of attention with respect to the existence and multiplicity of solutions. Since 2004, with a number of papers, Bereanu and Mawhin have considered such problems with Dirichlet, Neumann or periodic boundary conditions (see [1-4] and the references therein). In these papers, the various boundary value problems are reduced to the search for fixed points of some nonlinear operators defined on Banach spaces. In particular, they have studied some boundary value problems with nonhomogeneous boundary conditions and obtained the existence of solutions by the use of Schauder's fixed point theorem (see [3, 4]). Recently, 
Torres [5] has proved the existence of a solution of a forced Liénard differential equation with $\phi$-Laplacian by means of Schauder's fixed point theorem.

However, many nonlinear differential equations need to seek the existence of positive solutions because the positive solutions are very meaningful. The existence of positive solutions for homogeneous and nonhomogeneous boundary value problems have been studied by several authors and many interesting results have been obtained (only to mention some of them, see [6-9], their references and the papers citing them). The problems with negative coefficients for the boundary conditions (see [10-12]) often occur in some heat flow problems, the deflection of a beam, and Floquet theory of the beam equation and have been considered by some experts (see [7, 8, 10-12]). If the coefficient takes a negative value, then it is sometimes difficult to find an appropriate cone to guarantee the existence of a positive solution of the corresponding differential equation. Comparing with the previous result [2-4], the cone may be smaller.

The purpose of this paper is to establish the criteria of the existence of a positive solution to the problem (1.1) by utilizing the Krasnosel'skii fixed point theorem, even if some of the $\alpha_{i}$ coefficients are negative. The method of proof is inspired by the ideas exposed in [3-5, $7,8]$. As we will see, our results are new, and the interesting points of those results are the following two aspects: (i) Some of the $\alpha_{i}$ coefficients appearing in (1.1) are allowed to take a negative value. (ii) The existence of a positive solution for the class of $\phi$-Laplacian differential equations with a nonhomogeneous boundary condition is proved. Notice that the existence of a positive solution for the class of $\phi$-Laplacian equations has been less studied in the related literature.

This paper is organized as follows. In Section 2, we give some lemmas, which play an important role in the proof of the main theorem. In Section 3, we obtain the existence of a positive solution to the problem (1.1). Moreover, two examples are also given to illustrate the main results.

\section{Preliminaries and lemmas}

Let $X$ denote the Banach space $C([0, T], \mathbb{R})$ of continuous functions endowed with the maximum norm $\|u\|=\max _{t \in[0, T]}|u(t)|$. Define a nonlinear operator $S: X \rightarrow X$ by

$$
\begin{aligned}
(S u)(t) & :=\sum_{i=1}^{k} \alpha_{i} u\left(\eta_{i}\right)+\int_{0}^{t} \phi^{-1}\left(\beta+\int_{s}^{T} h(\tau) f(u(\tau)) d \tau\right) d s \\
& =\omega(u)+\int_{0}^{t} \phi^{-1}\left(\beta+\int_{s}^{T} h(\tau) f(u(\tau)) d \tau\right) d s .
\end{aligned}
$$

Deriving on both sides of (2.1) leads to

$$
(S u)^{\prime}(t)=\phi^{-1}\left(\beta+\int_{t}^{T} h(s) f(u(s)) d s\right)
$$

i.e.,

$$
\phi\left((S u)^{\prime}(t)\right)=\beta+\int_{t}^{T} h(s) f(u(s)) d s .
$$


Again, deriving in (2.3) implies

$$
\left(\phi\left((S u)^{\prime}(t)\right)\right)^{\prime}=-h(t) f(u(t))
$$

Moreover, from (2.1) and (2.3), we get that $(S u)(0)=\omega(u)$ and $\phi\left((S u)^{\prime}(T)\right)=\beta$. Therefore, the existence of a solution for Eq. (1.1) is equivalent to seeking a fixed point of the nonlinear operator $S$.

For the sake of convenience, we give the following conditions.

(A) Denote $\Delta:=\left\{i \in I: \alpha_{i}<0\right\}$ and $\nabla:=\left\{i \in I: \alpha_{i}>0\right\}$, and $\alpha_{i}$ satisfies the conditions

$$
\sum_{i=1}^{k} \alpha_{i} \geq 0
$$

and

$$
\sum_{i \in \nabla} \alpha_{i}<1
$$

(F) The function $f: \mathbb{R}^{+} \rightarrow \mathbb{R}^{+}$is continuous and satisfies $M_{1} \leq f(u) \leq M_{2}$, for any $u \geq 0$, where $0<M_{1}<M_{2}$ are two constants.

(B) $\beta+M_{2} \int_{0}^{T} h(t) d t<b$ (for $\left.0<b<+\infty\right)$.

(H) There exists a $d \in(0, T)$ such that $h(d)>0$, and let the inequality

$$
\sum_{i \in \Delta} \alpha_{i} \int_{0}^{\eta_{i}} \phi^{-1}\left[\beta+M_{2} \int_{s}^{T} h(\tau) d \tau\right] d s+\sum_{i \in \nabla} \alpha_{i} \int_{0}^{\eta_{i}} \phi^{-1}\left[\beta+M_{1} \int_{s}^{T} h(\tau) d \tau\right] d s \geq 0
$$

be true.

For the unbounded $\phi$-Laplacian $(b=+\infty)$, we obtain the following results.

Lemma 1 Assume that the conditions $(\mathrm{F})$ and $(\mathrm{H})$ hold, $u(t) \geq 0, \omega(u) \geq 0$, and $0<a<T$. Then there exists a constant $\gamma \in(0,1)$ such that

$$
\min _{t \in[a, T]}(S u)(t) \geq \gamma\|S u\|
$$

Proof From the representation (2.2) and the conditions (F)-(H), we have

$$
(S u)^{\prime}(t)=\phi^{-1}\left(\beta+\int_{t}^{T} h(\tau) f(u(\tau)) d \tau\right) \geq 0
$$

Again since $\beta \geq 0$, we get that $\|S u\|=(S u)(T)$. Therefore, applying the condition (F) leads to

$$
\begin{aligned}
\|S u\| & =(S u)(T) \\
& =\omega(u)+\int_{0}^{T} \phi^{-1}\left(\beta+\int_{s}^{T} h(\tau) f(u(\tau)) d \tau\right) d s \\
& \leq \omega(u)+\int_{0}^{T} \phi^{-1}\left(\beta+M_{2} \int_{s}^{T} h(\tau) d \tau\right) d s,
\end{aligned}
$$


and

$$
\begin{aligned}
\min _{t \in[a, T]}(S u)(t) & =(S u)(a) \\
& =\omega(u)+\int_{0}^{a} \phi^{-1}\left(\beta+\int_{s}^{T} h(\tau) f(u(\tau)) d \tau\right) d s \\
& \geq \omega(u)+\int_{0}^{a} \phi^{-1}\left(\beta+M_{1} \int_{s}^{T} h(\tau) d \tau\right) d s \\
& \geq \gamma\|S u\|,
\end{aligned}
$$

where

$$
\gamma=\frac{\int_{0}^{a} \phi^{-1}\left(\beta+M_{1} \int_{s}^{T} h(\tau) d \tau\right) d s}{\int_{0}^{T} \phi^{-1}\left(\beta+M_{2} \int_{s}^{T} h(\tau) d \tau\right) d s} .
$$

This completes the proof.

Next, let us define a cone by

$$
\mathcal{P}:=\left\{u \in X: u \geq 0, \omega(u) \geq 0, \min _{t \in[a, T]} u(t) \geq \gamma\|u\|\right\}
$$

The definition of the cone is inspired by the results in $[7,8]$. To show our main results, the following lemma is essential.

Lemma 2 Let the conditions $(\mathrm{A}),(\mathrm{F})$, and $(\mathrm{H})$ hold and the nonlinear operator $S$ be defined by (2.1). Then $S: \mathcal{P} \rightarrow \mathcal{P}$.

Proof From the definition of the operator $S$, we find for any $u \in \mathcal{P}$ that

$$
(S u)(t)=\omega(u)+\int_{0}^{t} \phi^{-1}\left(\beta+\int_{s}^{T} h(\tau) f(u(\tau)) d \tau\right) d s \geq 0
$$

The conditions $(\mathrm{F})$ and $(\mathrm{H})$ yield

$$
\begin{aligned}
\omega(S u)= & \sum_{i=1}^{k} \alpha_{i}(S u)\left(\eta_{i}\right) \\
= & \sum_{i=1}^{k} \alpha_{i}\left\{\omega(u)+\int_{0}^{\eta_{i}} \phi^{-1}\left(\beta+\int_{s}^{T} h(\tau) f(u(\tau)) d \tau\right) d s\right\} \\
= & \omega(u) \cdot\left(\sum_{i=1}^{k} \alpha_{i}\right)+\sum_{i \in \Delta} \alpha_{i} \int_{0}^{\eta_{i}} \phi^{-1}\left(\beta+\int_{s}^{T} h(\tau) f(u(\tau)) d \tau\right) d s \\
& +\sum_{i \in \nabla} \alpha_{i} \int_{0}^{\eta_{i}} \phi^{-1}\left(\beta+\int_{s}^{T} h(\tau) f(u(\tau)) d \tau\right) d s \\
\geq & \sum_{i \in \Delta} \alpha_{i} \int_{0}^{\eta_{i}} \phi^{-1}\left(\beta+M_{2} \int_{s}^{T} h(\tau) d \tau\right) d s
\end{aligned}
$$




$$
+\sum_{i \in \nabla} \alpha_{i} \int_{0}^{\eta_{i}} \phi^{-1}\left(\beta+M_{1} \int_{s}^{T} h(\tau) d \tau\right) d s
$$

$\geq 0$.

Further, Lemma 1 shows

$$
\min _{t \in[a, T]}(S u)(t) \geq \gamma\|S u\|
$$

Consequently, we get that $S: \mathcal{P} \rightarrow \mathcal{P}$.

Remark 1 If the coefficients $\left\{\alpha_{i}: i \in I\right\}$ are nonnegative, then the conclusion in Lemma 2 also holds without the hypothesis $(\mathrm{H})$.

Lemma 3 If $0<b<+\infty$ and, in addition, the assumptions of Lemma 2 and the condition (B) are satisfied, then the conclusions of Lemma 1 and Lemma 2 hold.

Lemma 4 (See [13]) Let $X$ be a Banach space and $\mathcal{P} \subseteq X$ be a cone. Suppose that $\Omega_{1}$ and $\Omega_{2}$ are bounded open sets contained in $X$ such that $0 \in \Omega_{1}$ and $\bar{\Omega}_{1} \subseteq \Omega_{2}$. Suppose further that $S: \mathcal{P} \cap\left(\bar{\Omega}_{2} \backslash \Omega_{1}\right) \rightarrow \mathcal{P}$ is a completely continuous operator. If either

(i) $\|S u\| \leq\|u\|$ for $u \in \mathcal{P} \cap \partial \Omega_{1}$ and $\|S u\| \geq\|u\|$ for $u \in \mathcal{P} \cap \partial \Omega_{2}$ or

(ii) $\|S u\| \geq\|u\|$ for $u \in \mathcal{P} \cap \partial \Omega_{1}$ and $\|S u\| \leq\|u\|$ for $u \in \mathcal{P} \cap \partial \Omega_{2}$,

then $S$ has at least one fixed point in $\mathcal{P} \cap\left(\bar{\Omega}_{2} \backslash \Omega_{1}\right)$.

\section{The main result}

Theorem 1 Assume that the conditions (A), (F), and (H) hold and $b=+\infty$. Then Eq. (1.1) has at least one positive solution.

Proof Lemma 2 shows that $S: \mathcal{P} \rightarrow \mathcal{P}$. In addition, a standard argument involving the Arzela-Ascoli theorem implies that $S$ is a completely continuous operator.

Now, we choose a positive constant $r_{1}$ such that

$$
r_{1} \leq \int_{0}^{a} \phi^{-1}\left(\beta+M_{1} \int_{s}^{T} h(\tau) d \tau\right) d s
$$

and define $\Omega_{1}:=\left\{u \in X:\|u\|<r_{1}\right\}$. For any $u \in \mathcal{P} \cap \partial \Omega_{1}$, we get from the condition (F) that

$$
\begin{aligned}
\|S u\| & =(S u)(T)=\omega(u)+\int_{0}^{T} \phi^{-1}\left(\beta+\int_{s}^{T} h(\tau) f(u(\tau)) d \tau\right) d s \\
& \geq \int_{0}^{T} \phi^{-1}\left(\beta+\int_{s}^{T} h(\tau) f(u(\tau)) d \tau\right) d s \\
& \geq \int_{0}^{a} \phi^{-1}\left(\beta+M_{1} \int_{s}^{T} h(\tau) d \tau\right) d s \\
& \geq r_{1}=\|u\| .
\end{aligned}
$$

Thus, for any $u \in \mathcal{P} \cap \partial \Omega_{1}$, we find that

$$
\|S u\| \geq\|u\| .
$$


From the hypothesis (A), we can let $\sum_{i \in \nabla} \alpha_{i}=1-\varepsilon, \varepsilon \in(0,1)$. Next, we choose a positive constant $r_{2}$ such that

$$
r_{2}=\max \left\{\frac{1}{\varepsilon} \int_{0}^{T} \phi^{-1}\left(\beta+M_{2} \int_{s}^{T} h(\tau) d \tau\right) d s, \frac{r_{1}}{\varepsilon}\right\}
$$

and define $\Omega_{2}:=\left\{u \in X:\|u\|<r_{2}\right\}$. Clearly, for any $u \in \mathcal{P} \cap \partial \Omega_{2}$, we obtain

$$
\begin{aligned}
\|S u\| & =(S u)(T)=\omega(u)+\int_{0}^{T} \phi^{-1}\left(\beta+\int_{s}^{T} h(\tau) f(u(\tau)) d \tau\right) d s \\
& \leq \sum_{i \in \nabla} \alpha_{i}\|u\|+\int_{0}^{T} \phi^{-1}\left(\beta+M_{2} \int_{s}^{T} h(\tau) d \tau\right) d s \\
& \leq \sum_{i \in \nabla} \alpha_{i}\|u\|+\varepsilon r_{2} \\
& =r_{2}\left[\sum_{i \in \nabla} \alpha_{i}+\varepsilon\right]=r_{2}=\|u\| .
\end{aligned}
$$

Then, for any $u \in \mathcal{P} \cap \partial \Omega_{2}$, it implies that

$$
\|S u\| \leq\|u\|
$$

Based on Lemma 4, we get from (3.1) and (3.2) that the operator $S$ has at least one fixed point. Thus, it follows that Eq. (1.1) has at least one positive solution.

Remark 2 If the coefficients $\left\{\alpha_{i}: i \in I\right\}$ are nonnegative, then the condition (A) is replaced with

$\left(\mathrm{A}^{\prime}\right) \quad \sum_{i=1}^{k} \alpha_{i} \in(0,1)$.

Applying the results in Remark 1 and Theorem 1, we get the following result.

Corollary 1 Assume that the conditions ( $\left.\mathrm{A}^{\prime}\right)$ and (F) hold and $b=+\infty$. Then Eq. (1.1) has at least one positive solution.

If $\phi: \mathbb{R} \rightarrow(-b, b)(0<b<+\infty)$, then we have the following result.

Theorem 2 Assume that the conditions (A), (F), (B), and (H) hold. Then Eq. (1.1) has at least one positive solution.

Proof Using Lemma 3 and the proof of Theorem 1, we get that the conclusion holds.

Example 1 Consider the differential equation

$$
\left(\left|u^{\prime}(t)\right|^{2} u^{\prime}(t)\right)^{\prime}=-t\left(e^{-\sin u(t)}+\cos u^{2}(t)+5\right), \quad t \in(0,4)
$$

subjected to the boundary conditions

$$
u(0)=\frac{1}{8} u(1)-\frac{1}{4} u(2)+\frac{1}{2} u(3), \quad \phi\left(u^{\prime}(4)\right)=1 .
$$


Clearly, we find

$$
\begin{aligned}
& \phi=\phi_{4}, \quad \text { is one } p \text {-Laplacian operator, } p=4, \\
& h(t)=t, \quad f(u)=e^{-\sin u}+\cos u^{2}+5 \\
& \omega(u)=\frac{1}{8} u(1)-\frac{1}{4} u(2)+\frac{1}{2} u(3), \\
& \alpha_{1}=\frac{1}{8}, \quad \alpha_{2}=-\frac{1}{4}, \quad \alpha_{3}=\frac{1}{2}, \quad \sum_{i=1}^{3} \alpha_{i}=\frac{3}{8} \quad \text { and } \quad \alpha_{1}+\alpha_{3}=\frac{5}{8} .
\end{aligned}
$$

Computing yields

$$
\begin{aligned}
& 4+\frac{1}{e} \leq f(u) \leq 6+e, \\
& -\frac{1}{4} \int_{0}^{2} \phi^{-1}\left[1+(6+e) \int_{s}^{4} \tau d \tau\right] d s+\frac{1}{8} \int_{0}^{1} \phi^{-1}\left[1+\left(4+\frac{1}{e}\right) \int_{s}^{4} \tau d \tau\right] d s \\
& \quad+\frac{1}{2} \int_{0}^{3} \phi^{-1}\left[1+\left(4+\frac{1}{e}\right) \int_{s}^{4} \tau d \tau\right] d s \approx 86.65>0 .
\end{aligned}
$$

Therefore, we conclude from Theorem 1 that Eq. (3.3)-(3.4) has at least one positive solution.

Example 2 Consider the differential equation

$$
\left(\frac{u^{\prime}(t)}{\sqrt{1+\left(u^{\prime}(t)\right)^{2}}}\right)^{\prime}=-t^{4}(\sin u(t)+2), \quad t \in(0,1)
$$

subjected to the boundary conditions

$$
u(0)=-\frac{1}{40} u(0.2)+\frac{1}{4} u(0.3)+\frac{1}{2} u(0.7), \quad \phi\left(u^{\prime}(1)\right)=\frac{1}{4}
$$

Obviously, we obtain

$$
\begin{aligned}
& \phi(u)=\frac{u}{\sqrt{1+u^{2}}}, \\
& h(t)=t^{4}, \quad f(u)=\sin u(t)+2, \\
& \omega(u)=-\frac{1}{40} u(0.2)+\frac{1}{4} u(0.3)+\frac{1}{2} u(0.7), \\
& \alpha_{1}=-\frac{1}{40}, \quad \alpha_{2}=\frac{1}{4}, \quad \alpha_{3}=\frac{1}{2}, \quad \sum_{i=1}^{3} \alpha_{i}=\frac{29}{40} \quad \text { and } \quad \alpha_{2}+\alpha_{3}=\frac{3}{4} .
\end{aligned}
$$

It is easy to verify that the conditions $(\mathrm{B}),(\mathrm{F})$, and $(\mathrm{H})$ hold. Consequently, we get from Theorem 2 that the equation (3.5)-(3.6) has at least one positive solution. 
Authors' contributions

The authors typed, read and approved the final manuscript.

\section{Acknowledgements}

The authors would like to thank the anonymous referees very much for helpful comments and suggestions which led to the improvement of the presentation and quality of the work. The work was supported partly by NSFC of Tianyuan Youth Foundation (No.11126125), K.C. Wong Magna Fund of Ningbo University and Ningbo Natural Science Foundation (2012A610031).

Received: 10 June 2012 Accepted: 23 October 2012 Published: 12 November 2012

\section{References}

1. Bereanu, C, Mawhin, J: Nonlinear Neumann boundary-value problems with $\phi$-Laplacian operators. An. Univ. "Ovidius" Constanţa, Ser. Mat. 12, 73-82 (2004)

2. Bereanu, C, Mawhin, J: Existence and multiplicity results for some nonlinear equations with singular $\phi$-Laplacian. J. Differ. Equ. 243, 536-557 (2007)

3. Bereanu, C, Mawhin, J: Boundary value problems for some nonlinear systems with singular $\phi$-Laplacian. Fixed Point Theory Appl. 4, 57-75 (2008)

4. Bereanu, C, Mawhin, J: Nonhomogeneous boundary value problems for some nonlinear equations with singular $\phi$-Laplacian. J. Math. Anal. Appl. 352, 218-233 (2009)

5. Torres, PJ: Nondegeneracy of the periodically forced Liénard differential equation with $\phi$-Laplacian. Commun. Contemp. Math. 13, 283-292 (2011)

6. Wang, Y, Hou, C: Existence of multiple positive solutions for one-dimensional p-Laplacian. J. Math. Anal. Appl. 315 114-153 (2006)

7. Webb, JRL, Lan, KQ: Eigenvalue criteria for existence of multiple positive solutions of nonlinear boundary value problems of local and nonlocal type. Topol. Methods Nonlinear Anal. 27, 91-115 (2006)

8. Webb, JRL, Infante, G: Positive solutions of nonlocal boundary value problems: a unified approach. J. Lond. Math. Soc. 74, 673-693 (2006)

9. Rachůnková, I, Stanĕk, S, Tvrdý, M: Solvability of Nonlinear Singular Problems for Ordinary Differential Equations. Contemporary Mathematics and Its Applications, vol. 5 (2008)

10. Loud, WS: Self-adjoint multi-point boundary value problems. Pac. J. Math. 24, 303-317 (1968)

11. Jerome, JW: Linear self-adjoint multi-point boundary value problems and related approximation schemes. Numer. Math. 15, 433-449 (1970)

12. Behncke, H, Hinton, D: Two singular point linear Hamiltonian systems with an interface condition. Math. Nachr. 283, 365-378 (2010)

13. Krasnosel'skii, MA: Positive Solutions of Operator Equations. Noordhoff, Groningen (1964)

\section{Submit your manuscript to a SpringerOpen ${ }^{\circ}$ journal and benefit from:}

- Convenient online submission

Rigorous peer review

- Immediate publication on acceptance

- Open access: articles freely available online

- High visibility within the field

- Retaining the copyright to your article 